\title{
A NEW CONSTRUCTION OF THE UNSTABLE MANIFOLD FOR THE MEASURE-PRESERVING HÉNON MAP
}

\author{
ERIK JENSEN
}

(Communicated by Jane M. Hawkins)

\begin{abstract}
Let $H$ denote the measure-preserving Hénon map with the parameter $a>0$. The map $H$ has a hyperbolic fixed point $\boldsymbol{p}$. The main result of this paper is that the unstable mainfold of $\boldsymbol{p}$ is the iterated limit of a very simple set. Informally,

$$
W^{u}(\boldsymbol{p})=\lim _{n \rightarrow \infty} H^{n}(\mathcal{L})
$$

where $\mathcal{L}$ is the line $y=-x$ and $W^{u}(\boldsymbol{p})$ denotes the unstable manifold of $\boldsymbol{p}$.
\end{abstract}

\section{BACKGROUND MATERIAL}

The Hénon map, in its original form, is written as

$$
H_{\text {original }}\left(\begin{array}{l}
x \\
y
\end{array}\right)=\left(\begin{array}{c}
1+y-a x^{2} \\
b x
\end{array}\right)
$$

[H1] and has Jacobian

$$
\mathrm{D} H_{\text {original }}\left(\begin{array}{l}
x \\
y
\end{array}\right)=\left(\begin{array}{cc}
-2 a x & 1 \\
b & 0
\end{array}\right)
$$

so that $\operatorname{det} \mathrm{D} H_{\text {original }}=-b$.

Let $H$ denote the measure-preserving Hénon map. Then

$$
H\left(\begin{array}{l}
x \\
y
\end{array}\right)=\left(\begin{array}{c}
1+y-a x^{2} \\
-x
\end{array}\right) .
$$

The equations for the fixed points of $H$ are

$$
\begin{aligned}
& x=1+y-a x^{2}, \\
& y=-x .
\end{aligned}
$$

Solving these equations gives two fixed points:

$$
\begin{aligned}
& \boldsymbol{p}=\left(\begin{array}{l}
p_{1} \\
p_{2}
\end{array}\right)=\left(\begin{array}{c}
\frac{1}{a}(-1-\sqrt{1+a}) \\
\frac{1}{a}(1+\sqrt{1+a})
\end{array}\right), \\
& \boldsymbol{q}=\left(\begin{array}{c}
q_{1} \\
q_{2}
\end{array}\right)=\left(\begin{array}{c}
\frac{1}{a}(-1+\sqrt{1+a}) \\
\frac{1}{a}(1-\sqrt{1+a})
\end{array}\right) .
\end{aligned}
$$

Received by the editors June 12, 2006 and, in revised form, September 29, 2006.

2000 Mathematics Subject Classification. Primary 37D10.

The author would like to thank Leo Jonker for his helpful suggestions. 
To see that $\boldsymbol{p}$ is hyperbolic, note that

$$
\mathrm{D} H(\boldsymbol{p})=\left(\begin{array}{cc}
-2 a p_{1} & 1 \\
-1 & 0
\end{array}\right)
$$

and has characteristic polynimial

$$
\lambda^{2}+2 a p_{1}+1
$$

so that the eigenvalues of $\mathrm{D} H(\boldsymbol{p})$ are

$$
\begin{aligned}
\lambda & =-a p_{1} \pm \sqrt{a^{2} p_{1}^{2}-1} \\
& =1+\sqrt{1+a} \pm \sqrt{1+a+2 \sqrt{1+a}}
\end{aligned}
$$

so that $\boldsymbol{p}$ is hyperbolic for $a>-1$.

The map $H$ is the composition of two involutions. This fact is very important, since it will give us a geometric picture of $H$. Let $\mathcal{L}$ denote the line $y=-x$, and let $\mathcal{T}_{\mathcal{L}}$ denote a perpendicular reflection in $\mathcal{L}$. Let $\mathcal{P}$ denote the parabola $x=\frac{1}{2}\left(1-a y^{2}\right)$, and let $\mathcal{T}_{\mathcal{P}}$ denote a horizontal reflection in $\mathcal{P}$. Then

$$
\begin{aligned}
& \mathcal{T}_{\mathcal{L}}\left(\begin{array}{l}
x \\
y
\end{array}\right)=\left(\begin{array}{c}
-y \\
-x
\end{array}\right) \\
& \mathcal{T}_{\mathcal{P}}\left(\begin{array}{l}
x \\
y
\end{array}\right)=\left(\begin{array}{c}
1-x-a y^{2} \\
y
\end{array}\right),
\end{aligned}
$$

and

$$
H=\mathcal{T}_{\mathcal{L}} \circ \mathcal{T}_{\mathcal{P}}
$$

Figure 1 is a diagram of the sets $\mathcal{L}$ and $\mathcal{P}$ for $a>0$.

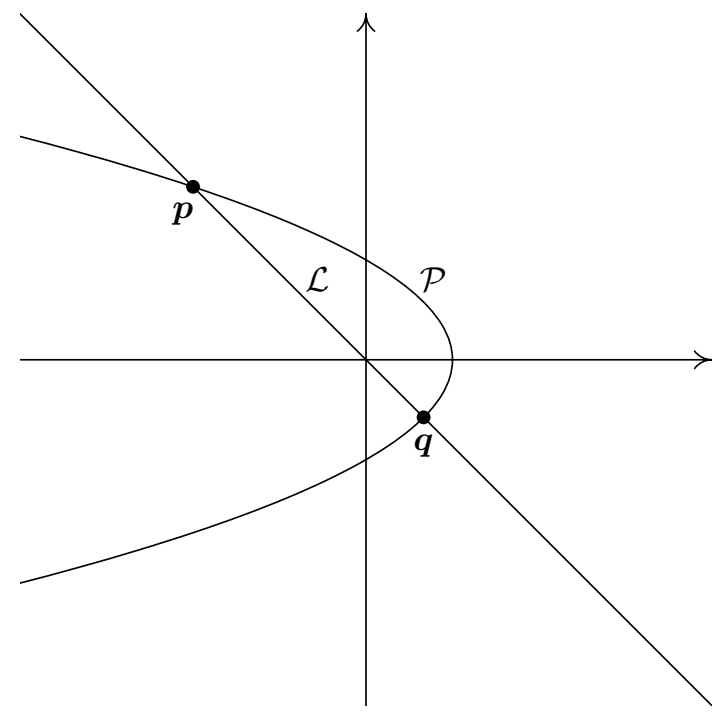

Figure 1. The map $H$ is a perpendicular reflection in $\mathcal{L}$ followed by a horizontal reflection in $\mathcal{P}$. 
Now, we calcuate the slope of the unstable manifold of $\boldsymbol{p}$ at $\boldsymbol{p}$. Let $s_{1}$ denote the expanding eigenvalue of $\mathrm{D} H(\boldsymbol{p})$, so that

$$
s_{1}=1+\sqrt{1+a}+\sqrt{1+a+\sqrt{1+a}} .
$$

Then

$$
\begin{aligned}
\operatorname{DH}(\boldsymbol{p})\left(\begin{array}{l}
x \\
y
\end{array}\right)=\left(\begin{array}{l}
s_{1} x \\
s_{1} y
\end{array}\right) & \Rightarrow\left(\begin{array}{cc}
-2 a p_{1} & 1 \\
-1 & 0
\end{array}\right)\left(\begin{array}{l}
x \\
y
\end{array}\right)=\left(\begin{array}{l}
s_{1} x \\
s_{1} y
\end{array}\right) \\
& \Rightarrow y=\frac{-1}{s_{1}} x
\end{aligned}
$$

so the slope of the unstable manifold of $\boldsymbol{p}$ at $\boldsymbol{p}$ is $\frac{-1}{s_{1}}$.

Let $C^{u}$ denote the branch of the unstable manifold of $\boldsymbol{p}$ that starts at $\boldsymbol{p}$ and initially moves to the right. Let $\boldsymbol{z}(t)=\left(z_{1}(t), z_{2}(t)\right)$ denote a parameterization of $C^{u}$.

\section{Motivation}

In 1995, Brown published a paper entitled 'Horseshoes in the measure-preserving Hénon map' [B]. In this paper, he proved that the measure-preserving Hénon map contains an embedded horseshoe for $a>0$. To do this, Brown used geometric methods to prove that $H$ has a homoclinic point for $a>0$, and then he used a contradiction argument to show that this homoclinic point must be transverse. By a theorem of Smale [S1], a transverse homoclinic point implies the existence of a horseshoe.

The main result of the current paper was motivated in large part by the geometric methods Brown used in his proof. Specifically, Brown used the fact that $C^{u}$ cannot cross $\mathcal{L}_{1}$ before crossing $\mathcal{L}_{0}$, where $\mathcal{L}_{n}$ denotes $H^{n}(\mathcal{L})$. We will prove this fact in Lemma 1.

Figure 2 shows the shape of $C^{u}$.

The point $\chi_{c}$ in Figure 2 is the intersection of $\mathcal{L}_{0}$ and $C^{u}$. By a result of Devaney [D1], $\chi_{c}$ must be a homoclinic point. Devaney, in his 1984 paper 'Homoclinic bifurcations and the area-conserving Hénon mapping' [D1], introduced the concept

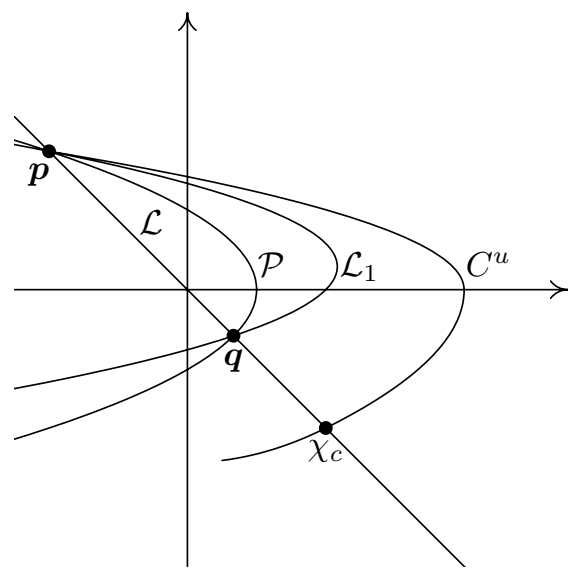

Figure 2. $\mathcal{L}, \mathcal{P}, \mathcal{L}_{1}, C^{u}$, and $\chi_{c}$ 
of $T$-reversibility. For our purposes, the Hénon map is $\mathcal{T}_{\mathcal{L}}$-reversible and $\boldsymbol{p} \in \mathcal{L}$, so Devaney's result implies that $W^{s}(\boldsymbol{p})=\mathcal{T}_{\mathcal{L}}\left(W^{u}(\boldsymbol{p})\right)$, where $W^{s}(\boldsymbol{p})$ denotes the stable manifold of $\boldsymbol{p}$ and $W^{u}(\boldsymbol{p})$ denotes the unstable manifold of $\boldsymbol{p}$. Since $\mathcal{T}_{\mathcal{L}}$ is a reflection in the line $\mathcal{L}$, then any point in $\mathcal{L} \cap W^{u}(\boldsymbol{p})$ must be in $W^{s}(\boldsymbol{p}) \cap W^{u}(\boldsymbol{p})$.

Brown used geometric methods to prove that $C^{u}$ crosses $\mathcal{L}$. For the purposes of the current paper, the most important of these is the following lemma:

Lemma 1. $C^{u}$ cannot cross $H(\mathcal{L})$ before crossing $\mathcal{L}$.

Proof. Suppose that $C^{u}$ crosses $H(\mathcal{L})$ at $\boldsymbol{r}$ before crossing $\mathcal{L}$. Then $H^{-1}(\boldsymbol{r}) \in \mathcal{L}$ and $H^{-1}(\boldsymbol{r}) \in C^{u}$, and $H^{-1}(\boldsymbol{r})$ precedes $\boldsymbol{r}$ on $C^{u}$. This contradiction proves the lemma.

From Figure 2, one can see that the previous lemma gives a rough picture of the shape of $C^{u}$. This is the observation that led to the result of the current paper. Let $\mathcal{L}_{n}$ denote $H^{n}(\mathcal{L})$; Figure 3 is a graph of $\mathcal{L}_{0}, \mathcal{L}_{1}$, and $\mathcal{L}_{2}$.

In Brown's proof, the set $\mathcal{L}_{1}$ played an important role in the proof that $H$ has a homoclinic point. The fact that $C^{u}$ could not cross $\mathcal{L}_{1}$ before crossing $\mathcal{L}_{0}$ gave us a rough picture of the shape of $C^{u}$. Extending this idea, we find that $C^{u}$ cannot cross $\mathcal{L}_{n}$ before crossing $\mathcal{L}_{n-1}$. It seems reasonable to investigate the possibility that each successive $\mathcal{L}_{n}$ is a better approximation of $C^{u}$. In fact, this is true. Further, one may obtain, in a precise way, a set $\mathcal{L}_{\infty}$ as a limit of the $\mathcal{L}_{n}$, with $\mathcal{L}_{\infty}=C^{u}$ between $\boldsymbol{p}$ and $\chi_{c}$.

So, we state the following theorem:

\section{Theorem 1.}

$$
\mathcal{L}_{\infty}=C^{u}
$$

between $\boldsymbol{p}$ and $\chi_{c}$.

The next section is devoted to a proof.

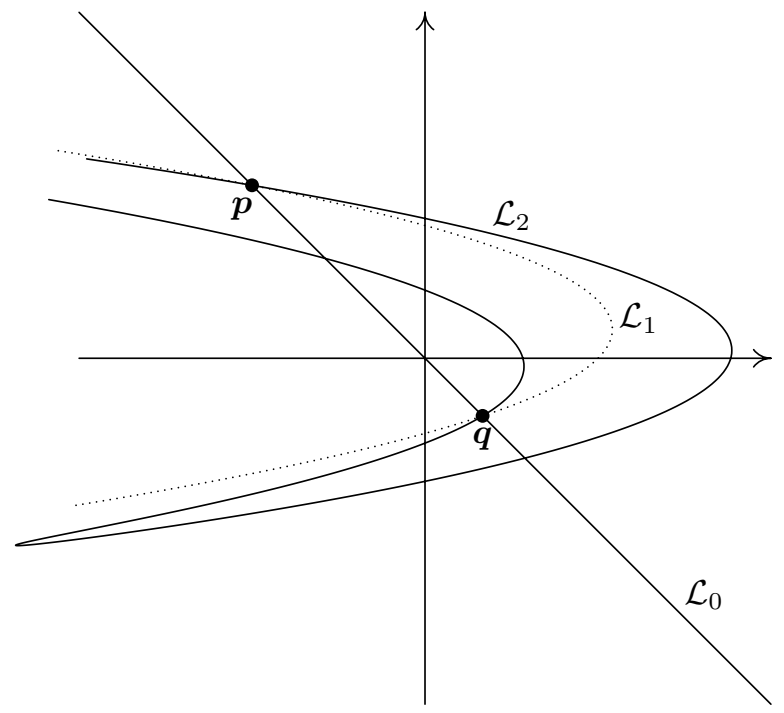

FiguRE $3 . \mathcal{L}_{0}, \mathcal{L}_{1}$, and $\mathcal{L}_{2}$ 


\section{Proof}

Theorem 1 can be proved via the following five steps:

(1) Show that $\mathcal{L}_{n} \cap \mathcal{L}_{n+1}=\{\boldsymbol{p}, \boldsymbol{q}\}$ for all $n$.

(2) Show that the slope of $\mathcal{L}_{n+1}$ at $\boldsymbol{p}$ is greater than the slope of $\mathcal{L}_{n}$ at $\boldsymbol{p}$ for all $n$.

(3) Show that there exists a set $\mathcal{J}$ such that $\widetilde{\mathcal{L}_{n}}$ is in the region bounded by $\mathcal{J}$ and $\mathcal{L}_{0}$ for all $n$, where $\widetilde{\mathcal{L}_{n}}$ is the segment of $\mathcal{L}_{n}$ from $\boldsymbol{p}$ to the first intersection of $\mathcal{L}_{n}$ with $\mathcal{L}_{0}$. This is illustrated in Figure 4.

(4) Define $\mathcal{L}_{\infty}$ in a precise way as a limit of the sets $\widetilde{\mathcal{L}_{n}}$.

(5) Show that $\mathcal{L}_{\infty}=C^{u}$ between $\boldsymbol{p}$ and $\chi_{c}$.

Step 1. Suppose that $\boldsymbol{v} \in \mathcal{L}_{n} \cap \mathcal{L}_{n+1}$. Then $H^{-n}(\boldsymbol{v}) \in \mathcal{L}_{0} \cap \mathcal{L}_{1}$, and so either $H^{-n}(\boldsymbol{v})=\boldsymbol{p}$, in which case $\boldsymbol{v}=\boldsymbol{p}$, or $H^{-n}(\boldsymbol{v})=\boldsymbol{q}$, in which case $\boldsymbol{v}=\boldsymbol{q}$. So, $\mathcal{L}_{n}$ and $\mathcal{L}_{n+1}$ intersect only at $\boldsymbol{p}$ and $\boldsymbol{q}$. Combining this fact with the result of Step 2 proves that $\widetilde{\mathcal{L}_{n+1}}$ is completely outside of $\widetilde{\mathcal{L}_{n}}$ for all $n$.

One should note that $\mathcal{L}_{n}$ and $\mathcal{L}_{n+2}$ do intersect at points other than $\boldsymbol{p}$ and $\boldsymbol{q}$.

Step 2. Define

$$
\begin{aligned}
& T_{0}(t)=t \\
& T_{1}(t)=t \\
& T_{n}(t)=1-T_{n-2}(t)-a\left[T_{n-1}(t)\right]^{2} \quad \text { for } n \geq 2 .
\end{aligned}
$$

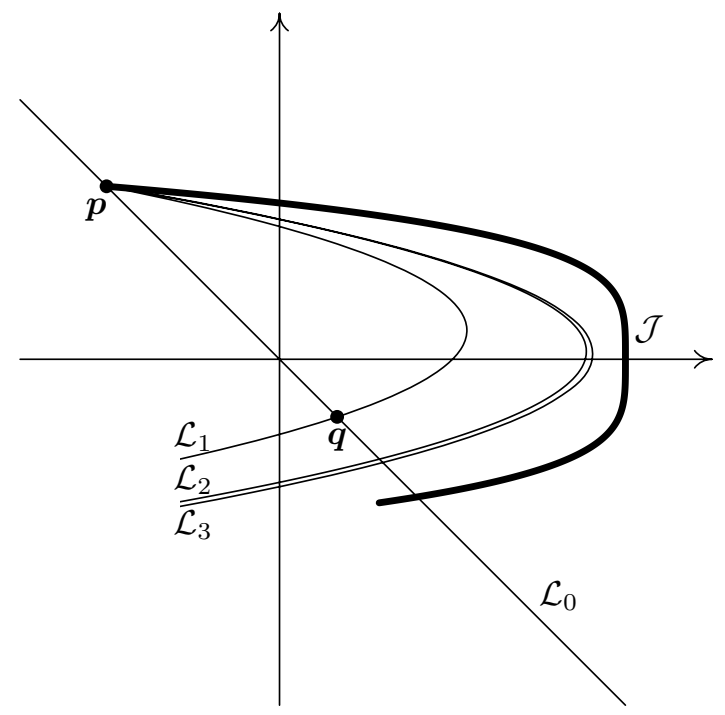

FiguRE 4 . What an outer bound $\mathcal{J}$ for the $\mathcal{L}_{n}$ might look like 
Then

$$
\begin{aligned}
\mathcal{L}_{0}(t) & =\left(\begin{array}{c}
T_{1}(t) \\
-T_{0}(t)
\end{array}\right), \\
\mathcal{L}_{1}(t) & =\left(\begin{array}{c}
T_{2}(t) \\
-T_{1}(t)
\end{array}\right), \\
\mathcal{L}_{n}(t) & =\left(\begin{array}{c}
T_{n+1}(t) \\
-T_{n}(t)
\end{array}\right) .
\end{aligned}
$$

Let $m_{n}(t)$ be the slope of $\mathcal{L}_{n}(t)$. Then

$$
m_{n}(t)=\frac{-\dot{T}_{n}(t)}{\dot{T}_{n+1}(t)} .
$$

The $m_{n}(t)$ can be expressed recursively as

$$
\begin{aligned}
m_{n}(t) & =\frac{-\dot{T}_{n}(t)}{\dot{T}_{n+1}(t)} \\
& =\frac{-\dot{T}_{n}(t)}{-\dot{T}_{n-1}(t)-2 a \dot{T}_{n}(t) T_{n}(t)} \\
& =\frac{1}{2 a T_{n}(t)+\frac{\dot{T}_{n-1}(t)}{\dot{T}_{n}(t)}} \\
& =\frac{1}{2 a T_{n}(t)-m_{n-1}(t)} .
\end{aligned}
$$

Now we may prove that the slope of $\mathcal{L}_{n+1}$ at $\boldsymbol{p}$ is greater than the slope of $\mathcal{L}_{n}$ at $\boldsymbol{p}$ for all $n$. It is important to note that $T_{n}\left(p_{1}\right)=p_{1}$ for all $n$.

We have that

$$
\begin{aligned}
& m_{0}\left(p_{1}\right)=-1, \\
& m_{1}\left(p_{1}\right)=\frac{1}{2 a T_{1}\left(p_{1}\right)+1}=\frac{1}{-1-2 \sqrt{1+a}}>-1 .
\end{aligned}
$$

We proceed by induction on $n$ :

$$
\begin{array}{rlrl}
m_{n}\left(p_{1}\right) & >m_{n-1}\left(p_{1}\right) \\
& \Longleftrightarrow & -m_{n}\left(p_{1}\right) & <-m_{n-1}\left(p_{1}\right) \\
& \Longleftrightarrow & 2 a p_{1}-m_{n}\left(p_{1}\right) & <2 a p_{1}-m_{n-1}\left(p_{1}\right) \\
& \Longleftrightarrow & \frac{1}{2 a p_{1}-m_{n}\left(p_{1}\right)} & >\frac{1}{2 a p_{1}-m_{n-1}\left(p_{1}\right)} \\
& \Longleftrightarrow & \frac{1}{2 a T_{n+1}\left(p_{1}\right)-m_{n}\left(p_{1}\right)} & >\frac{1}{2 a T_{n}\left(p_{1}\right)-m_{n-1}\left(p_{1}\right)} \\
m_{n+1}\left(p_{1}\right) & >m_{n}\left(p_{1}\right) .
\end{array}
$$

In order to justify the operation between lines 3 and 4 in the above inequalities, we must show that $2 a p_{1}-m_{n}\left(p_{1}\right)$ and $2 a p_{1}-m_{n-1}\left(p_{1}\right)$ have the same sign. As a consequence of the proof of Step 3, we have that

$$
0>m_{n}\left(p_{1}\right)>m_{n-1}\left(p_{1}\right)>-1
$$


which implies that

$$
2 a p_{1}<2 a p_{1}-m_{n}\left(p_{1}\right)<2 a p_{1}-m_{n-1}\left(p_{1}\right)<2 a p_{1}+1 \text {. }
$$

Since $2 a p_{1}$ and $2 a p_{1}+1$ are both negative, then $2 a p_{1}-m_{n}\left(p_{1}\right)$ and $2 a p_{1}-m_{n-1}\left(p_{1}\right)$ are both negative.

So, $m_{n+1}\left(p_{1}\right)>m_{n}\left(p_{1}\right)$ for all $n$.

Step 3. Figure 4 is an illustration of what a set $\mathcal{J}$ might look like.

We will show that $C^{u}$ is an appropriate $\mathcal{J}$. First, note that the slope of $C^{u}$ at $\boldsymbol{p}$ is greater than the slope of $\mathcal{L}_{1}$ at $\boldsymbol{p}$, so that $C^{u}$ starts above $\mathcal{L}_{1}$. If $C^{u}$ is not above all of the $\mathcal{L}_{n}$, then it must start between two consecutive $\mathcal{L}_{n}$. So, suppose that $C^{u}$ starts between $\mathcal{L}_{n}$ and $\mathcal{L}_{n+1}$. This leads to a contradiction as follows. Let $\boldsymbol{v} \in C^{u}$. Since $\boldsymbol{v}$ is between $\mathcal{L}_{n}$ and $\mathcal{L}_{n+1}$, then $H^{-1}(\boldsymbol{v})$ is between $\mathcal{L}_{n-1}$ and $\mathcal{L}_{n}$. But, since $\boldsymbol{v} \in C^{u}$, then $H^{-1}(\boldsymbol{v}) \in C^{u}$, and so $H^{-1}(\boldsymbol{v})$ is between $\mathcal{L}_{n}$ and $\mathcal{L}_{n+1}$. This is a contradiction, and so $C^{u}$ must be above $\mathcal{L}_{n}$ for all $n$.

Since $C^{u}$ crosses $\mathcal{L}_{0}$ (for a proof of this see [B] or [D1]), the previous argument also shows that all the $\widetilde{\mathcal{L}_{n}}$ are bounded.

Step 4. Now we define the set $\mathcal{L}_{\infty}$. First, let $\mathcal{V}_{\theta}$ be a ray from the origin with angle $\theta$, where $\theta \in\left[-\frac{\pi}{4}, \frac{3 \pi}{4}\right]$. Then, let $\theta_{n}=\widetilde{\mathcal{L}_{n}} \cap \mathcal{V}_{\theta}$. We know that $\lim _{n \rightarrow \infty} \theta_{n}$ exists since the sequence $\left\{\theta_{n}\right\}$ is strictly increasing and bounded. So, let

$$
\theta_{\infty}=\lim _{n \rightarrow \infty} \theta_{n}
$$

Then $\mathcal{L}_{\infty}$ is defined by

$$
\mathcal{L}_{\infty}=\left\{\theta_{\infty}: \theta \in\left[-\frac{\pi}{4}, \frac{3 \pi}{4}\right]\right\}
$$

This construction is illustrated in Figure 5.

Step 5. Let $\boldsymbol{v} \in \mathcal{L}_{\infty}$. We must show that $\lim _{n \rightarrow \infty} H^{-n}(\boldsymbol{v})=\boldsymbol{p}$.

We start with some definitions.

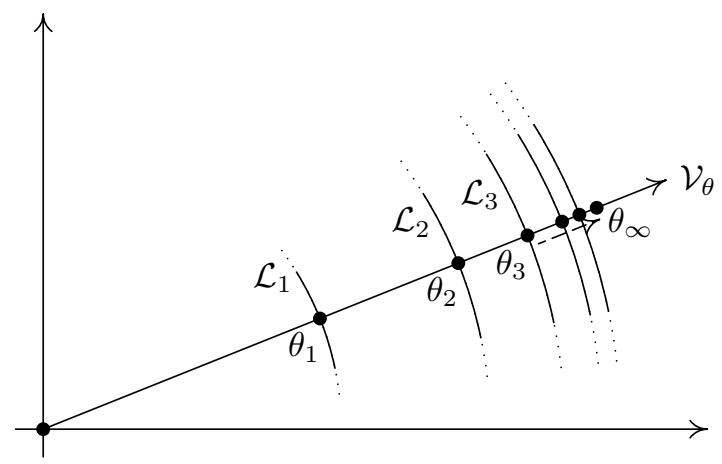

FiguRE 5. The construction of $\theta_{\infty}$ 


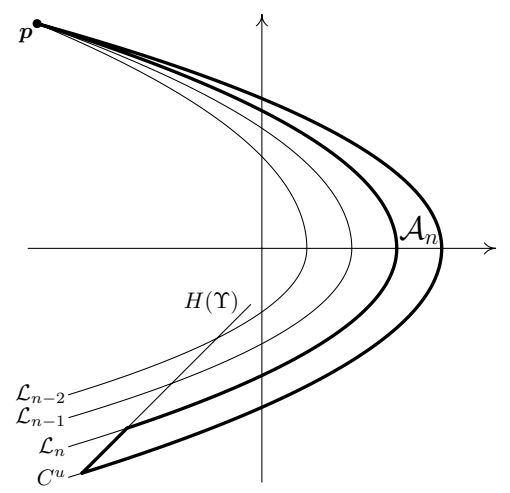

Figure 6 . The set $\mathcal{A}_{n}$ is bounded by $\mathcal{L}_{n}, H(\Upsilon)$, and $C^{u}$

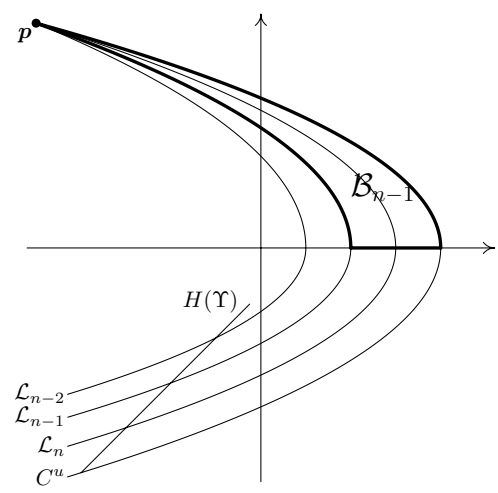

FiguRE 7 . The set $\mathcal{B}_{n-1}$ is bounded by $\mathcal{L}_{n-1}, \Upsilon$, and $C^{u}$

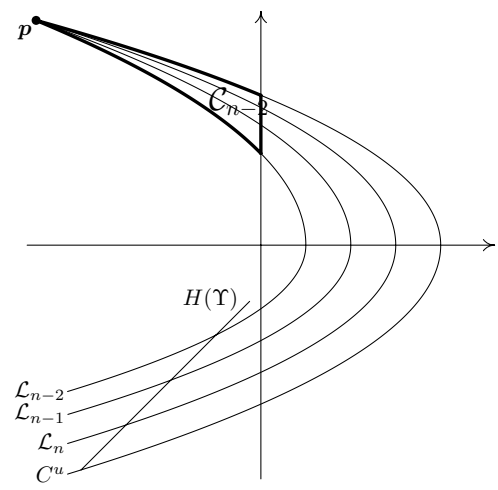

Figure 8 . The set $\mathcal{C}_{n-2}$ is bounded by $\mathcal{L}_{n-2}$, the positive vertical axis, and $C^{u}$

Let $\Upsilon$ be the line from the first intersection of $\mathcal{L}_{1}$ with the $x$-axis to the first intersection of $C^{u}$ with the $x$-axis. So, $\Upsilon$ is the line from $(1,0)$ to $\left(x_{+}, 0\right)$. Consider 
the image of $\Upsilon$ under $H$.

$$
H(\Upsilon)=H\left(\begin{array}{l}
t \\
0
\end{array}\right)=\left(\begin{array}{c}
1-a t^{2} \\
-t
\end{array}\right) \quad \text { for } t \in\left[1, x_{+}\right] .
$$

We will show that $H(\Upsilon)$ is below the line $\mathcal{L}_{0}$.

$$
-t<-1+a t^{2} \Longleftrightarrow 0<a t^{2}+t-1
$$

but $a t^{2}+t-1$ is an increasing function for $t \geq 1$, and $a \cdot 1^{2}+1-1=a>0$. So, $H(\Upsilon)$ is below the line $\mathcal{L}_{0}$.

Now we define the following sets:

$\mathcal{A}_{n}$ : the area bounded by $\mathcal{L}_{n}, H(\Upsilon)$, and $C^{u}$,

$\mathcal{B}_{n}$ : the area bounded by $\mathcal{L}_{n}, \Upsilon$, and $C^{u}$,

$\mathcal{C}_{n}$ : the area bounded by $\mathcal{L}_{n}$, the positive $y$-axis, and $C^{u}$.

These sets are illustrated in Figures 6, 7, and 8.

We note that

$$
\begin{array}{ll}
H^{-1}\left(\mathcal{A}_{n}\right) \subset \mathcal{B}_{n-1} & \text { for all } n, \\
H^{-2}\left(\mathcal{A}_{n}\right) \subset \mathcal{C}_{n-2} & \text { for all } n
\end{array}
$$

Now, define

$$
\begin{aligned}
& \mathcal{A}_{\infty}=\bigcap_{n=1}^{\infty} \mathcal{A}_{n}, \\
& \mathcal{B}_{\infty}=\bigcap_{n=1}^{\infty} \mathcal{B}_{n}, \\
& \mathcal{C}_{\infty}=\bigcap_{n=1}^{\infty} \mathcal{C}_{n} .
\end{aligned}
$$

It is clear that $\mathcal{L}_{\infty} \subset \mathcal{A}_{\infty}$. We will show that for any $\boldsymbol{v} \in \mathcal{A}_{\infty}, \lim _{n \rightarrow \infty} H^{-n}(\boldsymbol{v})=\boldsymbol{p}$.

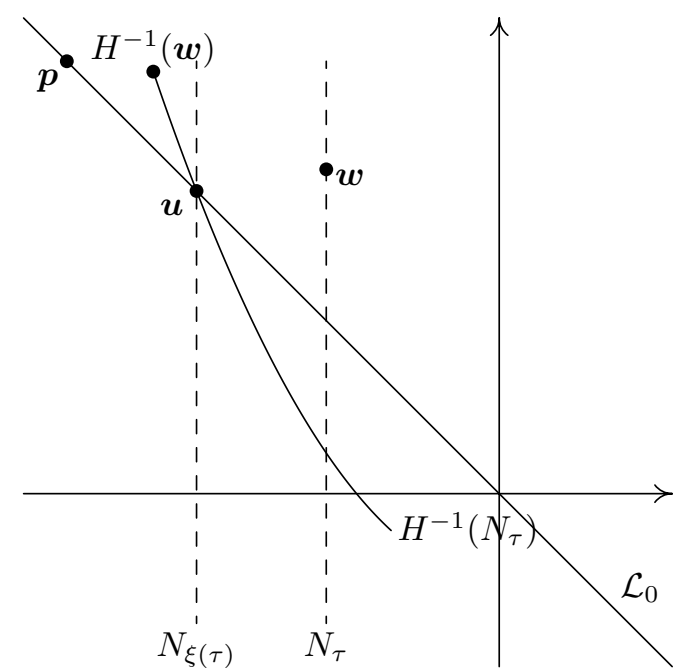

Figure 9. If $\boldsymbol{w} \in \mathcal{C}_{\infty}$, then $H^{-1}(\boldsymbol{w}) \in \mathcal{C}_{\infty}$ and $H^{-1}(\boldsymbol{w})$ is to the left of $\boldsymbol{w}$ 
First, note that since $\boldsymbol{v} \in \mathcal{A}_{\infty}$, then $\boldsymbol{v} \in \mathcal{A}_{n}$ for all $n$. We will show that $H^{-2-k}(\boldsymbol{v}) \in \mathcal{C}_{\infty}$ for all nonnegative integers $k$.

Since $\boldsymbol{v} \in \mathcal{A}_{n}$ for all $n$, then $H^{-2}(\boldsymbol{v}) \in \mathcal{C}_{n-2}$ for all $n$. So, $H^{-2}(\boldsymbol{v}) \in \mathcal{C}_{\infty}$.

Also, $H^{-1}\left(\mathcal{C}_{\infty}\right) \subset \mathcal{C}_{\infty}$, since

$$
\begin{aligned}
\boldsymbol{w} \in \mathcal{C}_{\infty} & \Rightarrow \boldsymbol{w} \in \mathcal{C}_{2} \cap \mathcal{C}_{3} \cap \mathcal{C}_{4} \cdots \\
& \Rightarrow H^{-1}(\boldsymbol{w}) \in \mathcal{C}_{1} \cap \mathcal{C}_{2} \cap \mathcal{C}_{3} \cdots \\
& \Rightarrow H^{-1}(\boldsymbol{w}) \in \mathcal{C}_{\infty}
\end{aligned}
$$

So, $H^{-2-k}(\boldsymbol{v}) \in \mathcal{C}_{\infty}$ for all nonnegative integers $k$.

Now, let $\boldsymbol{w}=H^{-2}(\boldsymbol{v})$, so that $\boldsymbol{w} \in \mathcal{C}_{\infty}$. Let $\tau$ be the $x$ co-ordinate of $\boldsymbol{w}$, and let $N_{\tau}$ denote the vertical line through $\boldsymbol{w}$. Consider Figure 9.

The map $H^{-1}$ maps $N_{\tau}$ to the parabola $y=a x^{2}-1+\tau$. Clearly, $H^{-1}(\boldsymbol{w})$ is to the left of the point $\boldsymbol{u}$, where $\boldsymbol{u}$ is the intersection of $\mathcal{L}_{0}$ and $H^{-1}\left(N_{\tau}\right)$.

By direct computation, the point $\boldsymbol{u}$ has $x$ co-ordinate

$$
-\frac{1}{2 a}-\frac{1}{2 a} \sqrt{1+4 a(1-\tau)}
$$

Let

$$
\xi(\tau)=-\frac{1}{2 a}-\frac{1}{2 a} \sqrt{1+4 a(1-\tau)} .
$$

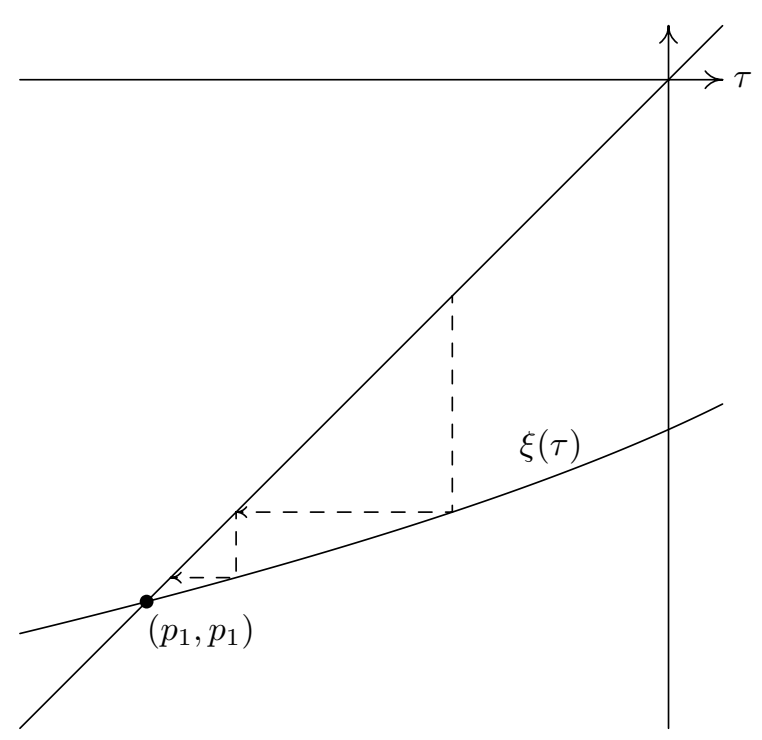

FiguRE 10. For $p_{1} \leq \tau \leq 0, \xi^{k}(\tau)$ approaches $p_{1}$ 
Then we have that

$$
\begin{aligned}
\boldsymbol{w} & \in \mathcal{C}_{\infty} \cap\{x \leq \tau\}, \\
H^{-1}(\boldsymbol{w}) & \in \mathcal{C}_{\infty} \cap\{x \leq \xi(\tau)\}, \\
H^{-2}(\boldsymbol{w}) & \in \mathcal{C}_{\infty} \cap\left\{x \leq \xi^{2}(\tau)\right\}, \\
\vdots & \\
H^{-k}(\boldsymbol{w}) & \in \mathcal{C}_{\infty} \cap\left\{x \leq \xi^{k}(\tau)\right\} .
\end{aligned}
$$

So, to complete the proof, we must show that $\lim _{k \rightarrow \infty} \xi^{k}(\tau)=p_{1}$. However, this is clear if we consider $\xi: \mathbb{R} \rightarrow \mathbb{R}$ as a dynamical system. The point $p_{1}$ is an attracting fixed point for this system, and all $\tau \in\left[p_{1}, 0\right]$ go to $p_{1}$ under iteration of $\xi$. This is shown in Figure 10.

So, we have shown that for $\boldsymbol{v} \in \mathcal{L}_{\infty}, \lim _{n \rightarrow \infty} H^{-n}(\boldsymbol{v})=\boldsymbol{p}$. Thus, $\mathcal{L}_{\infty}=C^{u}$ from $\boldsymbol{p}$ to $\chi_{c}$.

\section{Possible applications}

Theorem 1 gives a new way of looking at the unstable manifold. This result could have many applications in the field of chaotic dynamics, since a better understanding of the unstable manifold could lead to new techniques for finding homoclinic points.

In the following example, Theorem 1 is used to determine the slope of $C^{u}$ at $\boldsymbol{p}$ for the quadratic Hénon map $H$.

Example: Determining the slope of $C^{u}$ at $\boldsymbol{p}$. The slope of $C^{u}$ at $\boldsymbol{p}$ is the limit of the slopes of the $\mathcal{L}_{n}$ at $\boldsymbol{p}$.

Using the construction from Step 2 of the proof, one can express this limit as the following continued fraction:

$$
c=\frac{1}{2 a p_{1}-\frac{1}{2 a p_{1}-\frac{1}{2 a p_{1}-\cdots}}}
$$

Since $c=\frac{1}{2 a p_{1}-c}$, then

$$
\begin{aligned}
c & =\frac{2 a p_{1}-\sqrt{\left(2 a p_{1}\right)^{2}-4}}{2} \\
& =a p_{1}+\sqrt{a^{2} p_{1}^{2}-1} .
\end{aligned}
$$

Also, since $p_{1}=\frac{-1-\sqrt{1+a}}{a}$, then

$$
c=-1-\sqrt{1+a}+\sqrt{1+a+2 \sqrt{1+a}} .
$$

So, the slope of $C^{u}$ at $\boldsymbol{p}$ is $-1-\sqrt{1+a}+\sqrt{1+a+2 \sqrt{1+a}}$. 
In Section 1 , the slope $s_{1}$ of $C^{u}$ at $\boldsymbol{p}$ was determined using eigenvectors of the Jacobian. The result was

$$
s_{1}=\frac{-1}{1+\sqrt{1+a}+\sqrt{1+a+2 \sqrt{1+a}}} .
$$

To double check that $c=s_{1}$ :

$$
s_{1} \cdot \frac{1+\sqrt{1+a}-\sqrt{1+a+2 \sqrt{1+a}}}{1+\sqrt{1+a}-\sqrt{1+a+2 \sqrt{1+a}}}=c .
$$

\section{REFERENCES}

[ASY] K. Alligood, T. Sauer, and J. Yorke, Chaos: An Introduction to Dynamical Systems, Springer-Verlag, New York, 1996. MR1418166 (98a:58113)

[B-G] June Barrow-Green, Poincaré and the three body problem, American Mathematical Society, Providence, RI, 1997. MR1415387 (97g:01013)

[B] Ray Brown, Horseshoes in the measure preserving Hénon map, Ergod. Th. and Dyn. Sys. 15 (1995), 1045-1059. MR1366307 (96j:58135)

[DN] R. Devaney and Z. Nitecki, Shift automorphisms in the Hénon mapping, Comm. Math. Phys. 67 (1979), 137-148. MR539548 (80f:58035)

[D1] R. Devaney, Homoclinic bifurcations and the area-conserving Hénon mapping, J. Diff. Equ. 51 (1984), 254-266. MR731153 (85k:58054)

[D2] R. Devaney, An Introduction to Chaotic Dynamical Systems, Westview Press, Colorado, 1989. MR1046376 (91a:58114)

[G] James Gleick, Chaos: Making a New Science, Viking Press, New York, 1987. MR1010647 (91d:58152)

[H1] M. Hénon, A two dimensional mapping with a strange attractor, Comm. Math. Phys. 50 (1976), 65-80. MR0422932 (54:10917)

[H2] M. Hénon, Numerical study of quadratic area-preserving mappings, Quart. Appl. Math. XXVII(3) (1969), 291-312. MR0253513 (40:6727)

[J] Erik Jensen, Horseshoes in the measure preserving Hénon map for all even exponents, M. Sc. thesis, Queen's University, 2004.

[L] E. N. Lorenz, Deterministic non-periodic flow, J. Atmospheric Sci. 20 (1963), 130-141.

[S1] S. Smale, Diffeomorphisms with many periodic points. In 'Differential and Combinatorial Topology', pp. 63-80, Princeton University Press, Princeton, New Jersey, 1965. MR0182020 (31:6244)

[S2] S. Smale, Finding a horseshoe on the beaches of Rio, Math. Intelligencer 20(1) (1998), 39-44. MR1601831 (98i:58002)

Department of Mathematics and Statistics, Jeffery Hall, Queen's University, Kingston, Ontario, Canada K7L 3N6

E-mail address: jensene@mast.queensu.ca 